\title{
Benchmarking the MEKAL spectral code with solar X-ray spectra ${ }^{\star}$
}

\author{
K.J.H. Phillips ${ }^{1}$, R. Mewe ${ }^{2}$, L.K. Harra-Murnion ${ }^{3}$, J.S. Kaastra ${ }^{2}$, P. Beiersdorfer ${ }^{4}$, G.V. Brown ${ }^{4}$, and D.A. Liedahl ${ }^{4}$ \\ 1 Space Science Department, Rutherford Appleton Laboratory, Chilton, Didcot, Oxon. OX11 0QX, UK \\ 2 SRON Laboratory for Space Research, Sorbonnelaan 2, 3584 CA Utrecht, The Netherlands \\ 3 Mullard Space Science Laboratory, Holmbury St Mary, Dorking, Surrey RH5 6NT, UK \\ ${ }^{4}$ Dept. of Physics and Space Technology, Lawrence Livermore National Laboratory, 7000 East Avenue, Livermore, CA 94551, \\ U.S.A.
}

Received February 25; accepted May 20, 1999

\begin{abstract}
With the likelihood that high-resolution soft X-ray spectra of non-solar astronomical sources will soon become available, it is desirable to examine the accuracy of spectral synthesis codes. In this paper, a benchmark study of the MEKAL code, extensively used in the past for spectra from EUVE, $A S C A, S A X$, and other spacecraft, is presented using high-resolution solar flare X-ray spectra obtained with the Bragg Flat Crystal Spectrometer (FCS) on $S M M$. Lines in the range 5-20 $\AA$ are used to adjust the wavelengths in the MEKAL code. Many of the lines are due to Fe ions, and arise from $3-2$ transitions for spectra obtained during the decay phase of one of the flares, while others arise from higher-excitation $(4-2,5-2$ etc.) transitions for spectra obtained near the peak of a second flare. Laboratory measurements of the wavelengths of these lines were also used to confirm the $S M M$ values as well as published identifications from the HULLAC atomic code. The adjustments needed were up to $35 \mathrm{m \AA}$ for line wavelengths above $13 \AA$ but much less at shorter wavelengths. Some of these adjustments will be perceptible for spectra from the forthcoming $X M M$ and Chandra spacecraft.
\end{abstract}

Key words: atomic data - line: identification - sun: $\mathrm{X}$-rays, gamma-rays - X-rays: general

\section{Introduction}

Analysis of the soft X-ray and EUV spectra from non-solar astronomical sources in the past has generally made use of the several spectral synthesis codes that are available in the literature. The MEKAL code, used via the SPEX

Send offprint requests to: K.J.H. Phillips,

e-mail: K.J.H. Phillips@rl.ac.uk

* Appendices A and B are only available in electronic form at the http://www.edpsciences.com spectral software package (Mewe et al. 1995; Kaastra et al. 1996), has been one of the most frequently used of these, and contains a considerable amount of data relating to atomic transitions that give rise to both line and continuous spectra. The original MEKA code (Mewe et al. 1985; Kaastra \& Mewe 1993) was modified to allow for the fact that comparison of theoretical spectra with those observed by the $A S C A$ spacecraft of the central regions of galaxy clusters (Fabian et al. 1994) showed a discrepancy with the intensity ratio of the two groups of spectral lines due to $n=3-2$ and $n=4-2$ ( $n=$ principal quantum number) transitions in various ions of Fe (specifically FeXVII-FeXXIV). The addition of more than 2000 of the strongest lines from the Fe L complex in the $7-19 \AA$ range using data calculated with HULLAC (the Hebrew University/Lawrence Livermore Atomic Code) (e.g. Klapisch et al. 1977) has enabled this discrepancy to be largely removed (Liedahl et al. 1994, 1995). The latest version of the code was implemented as "MEKAL" (Mewe-Kaastra-Liedahl) in XSPEC (version 8.3) (cf. Mewe et al. 1995).

Previously, most spectra of non-solar sources have been of low resolution (e.g., $A S C A$ spectra have an energy resolution $\Delta E / E \approx 0.1$ at $1 \mathrm{keV})$, but with the imminent launch of the NASA spacecraft Advanced $X$-ray Astrophysics Facility or AXAF (now baptized as Chandra $X$-ray Observatory), and that of the ESA spacecraft $X$-ray Multi-Mirror Mission (XMM), we shall soon have available soft X-ray spectra with very high spectral resolution: the Low-Energy Transmission Grating Spectrometer (LETGS) $(4-155 \AA$ ) on Chandra, for example, will have a wavelength resolution of $\sim 50 \mathrm{m \AA}$ (e.g. Mewe et al. 1991). Also the Reflection Grating Spectrometer (RGS) $(5-35 \AA)$ on $X M M$ has a spectral resolution of about $50 \mathrm{~m} \AA$. For examples of simulated Chandra and XMM spectra see, e.g., Mewe (1991). 
This makes it much more important than in the past that accurate information about line excitation rates and wavelengths is contained in the spectral code.

Very high resolution soft X-ray spectra have been obtained in the past using crystal spectrometers dedicated to solar active regions and flares. Among these are spectra from the Flat Crystal Spectrometer (FCS), part of the X-ray Polychromator which was on board the Solar Maximum Mission spacecraft (SMM), which operated between 1980 and 1989. This paper is concerned with a benchmark study of the MEKAL code using solar flare spectra from the FCS instrument in the wavelength range $5-20 \AA$ and a spectral resolution of between 1 and $22 \mathrm{~m} \AA$. This wavelength range covers, for example, a large part of that of the RGS on XMM. In the following sections, we describe the FCS instrument and the data obtained from it, then discuss the fitting of two solar flare spectra chosen for detailed analysis to the MEKAL model. The adjustments to the atomic data included in MEKAL are outlined, as are the identifications of lines. Details are given in Appendices I and II to this paper. For the identifications, we used not only $S M M$ FCS data but data from highresolution spectra obtained from the Lawrence Livermore Electron Beam Ion Trap (EBIT) and the Princeton Large Torus (PLT) tokamak (also briefly described). We regard this analysis as a considerable advance in obtaining an improved spectral code, and we expect the code to contain much more precise data than before to enable satisfactory comparisons with Chandra and XMM spectra when they become available.

\section{SMM FCS solar flare spectra}

The $S M M$ spacecraft operated fully for nine months in 1980, from the time of launch (February) to the time when an attitude control unit on the spacecraft failed (November), and from April 1984 when Space Shuttle astronauts repaired the attitude control unit to December 1989 when the spacecraft re-entered the Earth's atmosphere. The FCS instrument was unable to operate while the attitude control unit was unavailable (November 1980 to April 1984). The full spectral range of the FCS was $1.5-20 \AA$ (in principle slightly longer wavelengths were accessible, but the sensitivity was too small to make spectra in this range useful). The instrument consisted of seven Bragg-diffracting crystals mounted on a single rotatable shaft, with solar X-rays incident on them via a fine collimator (FWHM response $14^{\prime \prime}$ ). X-ray photons were detected by proportional counters (one per crystal). The whole instrument could be moved in a scanning motion with the crystals fixed in wavelength at so-called "home position" spectral lines so that images of flares and active regions could be formed. Alternatively, with the collimator pointed at, e.g., the brightest point of a flare, the crystal shaft could be rotated through all or part of its range to obtain spectra in all seven channels (these are numbered 1 to 7 in decreasing order of wavelength). A summary of the spectroscopic results is given by Phillips (1999). During the first part of the mission, in 1980, when the Sun was near the peak of its activity cycle, the FCS was used largely as an imaging instrument, and relatively few flare spectra were obtained; only on one occasion were the FCS crystals driven over their entire range to get full spectral coverage. From 1984 to 1989, when the Sun was at first near minimum activity but later at a high state of activity, many spectra of active regions and flares were obtained, but by this time one then later other detectors had failed so that the spectral coverage was never complete.

Despite these operating problems, sets of spectra taken on two occasions are very suitable for analysis and comparison with MEKAL theoretical spectra. Those obtained during the decay of an M3 flare on 1980 August 25 form one of these sets. The spectral coverage is complete in this one case, but there is significant line emission only in the range $5-20 \AA$, covered by channels 1 to 4 . The scan was started at 13:10 U.T. and lasted approximately $17.5 \mathrm{~min}-$ utes, during which time the flare X-ray emission appreciably decreased. A notable feature of the spectra obtained is the large number of $\mathrm{Fe}$ ion lines in the $10.5-17 \AA$ range, mostly due to $n=3-2$ transitions in Fe XVII-Fe XIX. The analysis and a full line list with identifications are given by Phillips et al. (1982).

On the second occasion, several spectral scans were made during an M4.5 flare on 1985 July 2 between 21:19 U.T. and 21:41 U.T., which included the peak at about 21:25 U.T. As a consequence, much higher-excitation lines are apparent in these spectra, notably in the $7.3-10 \AA$ range which includes intense $n \geq 4-2$ lines in various Fe ions (mostly from FexIX to Fe XxIV). The analysis, in which several lines were identified for the first time, is described by Fawcett et al. (1987). Some of the FexxI and Fe XXII lines are density-dependent, and values obtained in an analysis of the spectra were discussed by Phillips et al. (1996b). The sequence of spectral scans was briefly as follows. The home position lines were first scanned, and having checked that the flux in them was large, the onboard software controlling the FCS commanded a spectral scan over the short-wavelength range. The home position line flux was again checked, then a second scan was made covering longer wavelengths, then a third and finally a fourth scan were made to cover the entire range of the FCS crystals. Four of the seven channels were operating at the time of this flare. The scans from channel 3, covering the wavelength range $7.33-10.09 \AA$ (with two small data drop-outs), are the ones discussed here and which include the high-excitation $\mathrm{Fe}$ ion lines.

As mentioned, the FCS had unprecedentedly good spectral resolution for the soft X-ray region, and even now there has not been a space-based instrument with better resolution except for limited spectral ranges. (Some spectrometers on plasma devices such as the PLT tokamak 

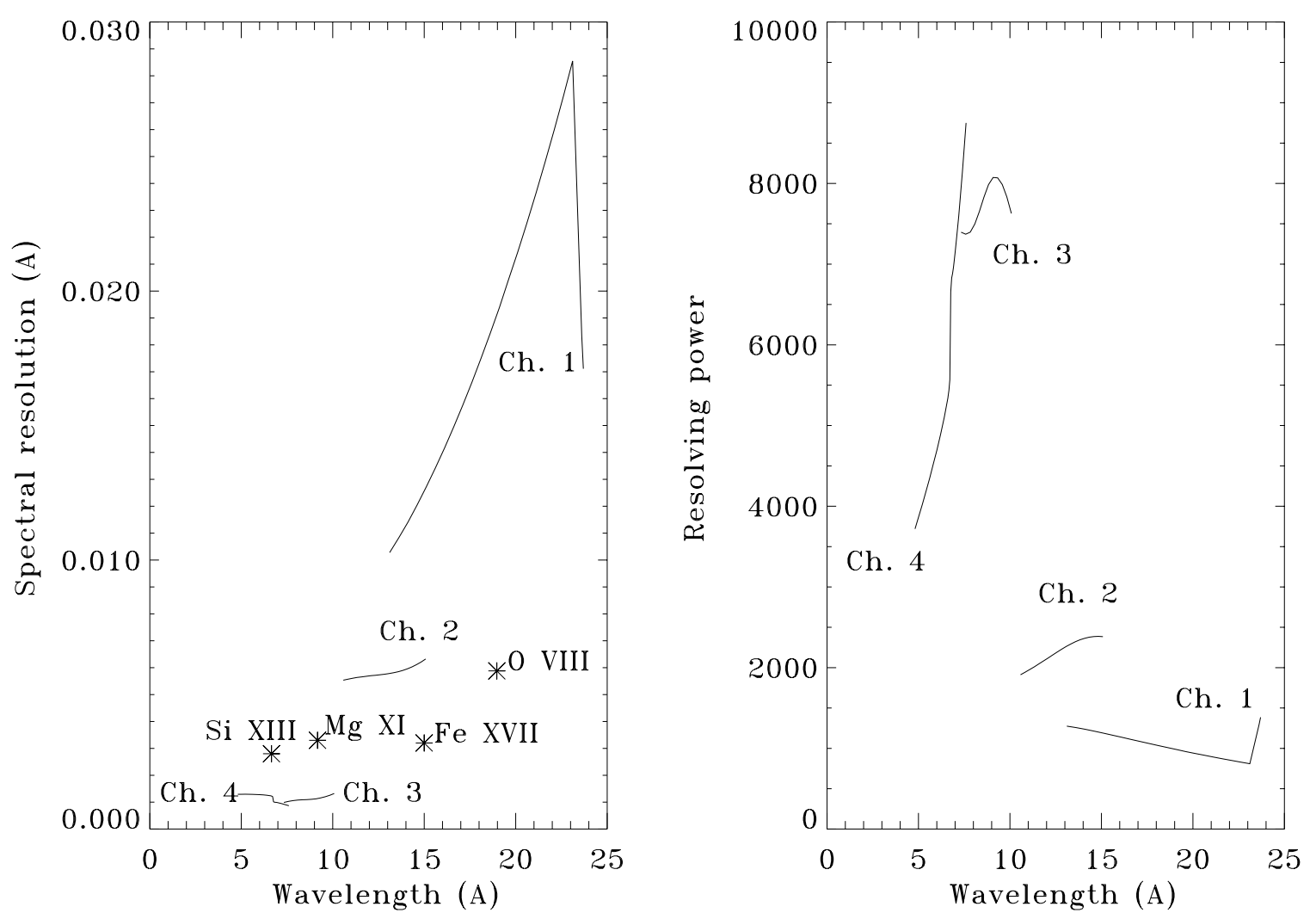

Fig. 1. a) Spectral resolution $\Delta \lambda(\mathrm{FWHM}$, in $\AA$ ) and b) resolving power $\lambda / \Delta \lambda$ of channels 1 to 4 of the FCS instrument, determined by the crystal rocking curve. The thermal Doppler line profile width (FWHM) of four representative lines in this range are individually plotted: the wavelengths are O viII $18.97 \AA$, Fe XVII $15.01 \AA$, Mg XI $9.17 \AA$, Si XIII $6.65 \AA$

do now have better resolution than the FCS.) The instrumental resolution of the FCS was determined largely by the width of the crystal rocking curve, a function of wavelength over the range of each crystal. As this is an important characteristic that determines the precision of line wavelengths, we plot both the FWHM $(\AA)$ of the rocking curve $\Delta \lambda$ (Fig. 1a) and the resolving power $\lambda / \Delta \lambda$ (Fig. 1b) as a function of wavelength. As can be seen, the resolving power of channels 1 and 2 is between 1000 and 2500, but is much greater for channels 3 and 4 covering shorter wavelengths. For channels 1 and 2, the instrumental width (FWHM) determined by the crystal rocking curve is much larger than the width (FWHM) of the thermal Doppler profile of spectral lines in this range. Examples of the latter, given by $\left.1.665 \times(\lambda / c) \sqrt{(} T_{\text {ion }} / m_{\text {ion }}\right)\left(\lambda\right.$ in $\AA, T_{\text {ion }}$ the ion temperature in $\mathrm{K}$ of peak line emissitivity, $m_{\text {ion }}$ the ion mass), are plotted in Fig. 1a. As can be seen, the thermal Doppler width is larger than the rocking curve width for channels 3 and 4 .

Plasma turbulence is a significant line broadening mechanism for flare spectra at the time of the flare impulsive (onset) phase, but is likely to be much smaller or nearly zero for later stages, as with the flare spectra we analyzed. In general we found that a combination of thermal
Doppler and the calculated FCS instrumental broadening adequately represented the observed line profiles.

Both the relative and absolute wavelength scale of FCS should be very precise. This is because of the highprecision Baldwin drive-encoder unit that was used for the FCS crystal drive. The output of this unit is crystal drive address rather than wavelength directly. An algorithm exists in the standard analysis software package (now incorporated in the SolarSoftWare or SSW system: see Freeland \& Handy 1998) for the conversion. The usual Bragg condition $N \lambda=2 d \sin \theta$ ( $N$ is the spectral order, generally 1 except for a few lines that are intense enough to be significant in second order) must be corrected for crystal refractive index and non-flatness. These were precisely measured before launch. The accuracy of relative wavelengths is estimated by Phillips et al. (1982) to be about $2 \mathrm{~m} \AA$ for $\lambda<15 \AA$ (i.e. over most of the range considered here). Absolute wavelengths were obtained using the home position lines in each of the seven channels as reference. For channel 1, this was the O VIII Ly- $\alpha$ line, but for the remaining channels the reference was the resonance line $\left(1 \mathrm{~s}^{2}{ }^{1} \mathrm{~S}_{0}-1 \mathrm{~s} 2 \mathrm{p}{ }^{1} \mathrm{P}_{1}\right)$ of various He-like ions. The theoretical wavelengths of these lines are known to better than $1 \mathrm{m \AA}$ so the absolute wavelengths should be 
accurate to 2 or $3 \mathrm{m \AA}$ over much of the FCS wavelength range used for this analysis. It should be noted, however, that many of the line wavelengths given by Phillips et al. above about $15 \AA$ are up to $5 \mathrm{~m} \AA$ larger measured against the wavelength scale in the more recent SSW software. A few wavelengths at shorter wavelengths have also been slightly adjusted (generally by not more than $1 \mathrm{m \AA}$ ).

The conversion of photon count rates in the FCS spectra to absolute intensity units (photons $\mathrm{cm}^{-2} \mathrm{~s}^{-1} \AA^{-1}$ ) is possible through pre-launch data referring to parameters such as measured crystal sizes, reflectivities etc. Again, standard software does this conversion (the procedure for the latter was described by Phillips et al. 1982). Since a large number of parameters are involved and the accuracy was impossible to check after $S M M$ was launched, the absolute intensities are probably not more accurate than $25 \%$.

With flare spectra from the FCS, there is a large background emission which is instrumental in origin and is much larger than the theoretical free-bound and free-free continuum emission. It is due to fluorescence by solar hard X-rays (with photon energies of $9 \mathrm{keV}$ or more) of the crystal material; the fluoresced radiation is emitted in all directions from each crystal, and so forms a continuous background when detected by the proportional counters. During the time it takes for the crystals to be scanned over their particular wavelength ranges (about $17.5 \mathrm{~min}-$ utes in the case of the August 25 flare), the flare intensity will have changed. This has two consequences important for this work. First, the radiation fluorescing the crystals varies with time, so that the background is strongly wavelength-dependent. Secondly, the line intensities will also be a function of time and therefore wavelength, so that the emission measure $E M=N_{\mathrm{e}}^{2} V$ as a function of temperature $T$ ( $N_{\mathrm{e}}$ is the electron density, $V$ the volume) will apparently vary, reflecting the state of the emitting plasma at the time of the scan.

Channels 1 and 2 of the FCS overlap in the region 13.10-14.94 $\AA$, a fact which is useful in comparing the line intensities and wavelength scales. As the wavelength range 13.10 - $14.94 \AA$ was observed in channel 1 soon after the start of the spectral scans during the 1980 August 25 flare but much later in channel 2 , we expect higher-ionization stages to be prominent in the channel 1 scan but less obvious in the channel 2 scan. This is well illustrated for the region $13.45-13.70 \AA$ which contains the well-known triplet of NeIX (due to $1 \mathrm{~s}^{2}{ }^{1} \mathrm{~S}_{0}-1 \mathrm{~s} 2 \mathrm{p}{ }^{1} \mathrm{P}_{1}, 1 \mathrm{~s} 2 \mathrm{p}{ }^{3} \mathrm{P}_{1,2}$, and $1 \mathrm{~s} 2 \mathrm{~s}{ }^{3} \mathrm{~S}_{1}$ transitions) as well as several Fe XIX lines of the $2 \mathrm{p}^{4}-2 \mathrm{p}^{3} 3 \mathrm{~d}$ array. Because Fe XIX is a hotter ion than NeIX, the Fe XIX lines are more intense relative to the Ne IX lines in the channel 1 scan than in the channel 2 scan. There are other more minor differences of a similar nature. The wavelength scales of the two channels are very slightly different over this range: channel 1 wavelengths are approximately $1-2 \mathrm{~m} \AA$ longer than those of channel 2 .
For the original analysis of the 1980 August 25 flare, Phillips et al. (1982) listed 205 recognizable line features, of which 165 were assigned identifications. The analysis of the 1985 July 2 flare by Fawcett et al. (1987) in the $7.85-10.0 \AA$ range resulted in a list of 47 recognizable line features, of which 38 were not in the list of Phillips et al. Identifications of these lines (which are mostly due to $\mathrm{L}$ complex lines with $n \geq 4-2$ transitions in various $\mathrm{Fe}$ ions) were assigned by comparison with data calculated from a Hartree-Fock code (Cowan 1981).

\section{Comparing FCS spectra with the MEKAL model}

\subsection{Fitting procedure}

We analyzed the two flare spectra by splitting the total range covered in each case into several smaller intervals. This facilitated the comparison with model spectra through the fact that the large instrumental background varies with wavelength in an irregular way over the whole range but over smaller ranges the variation can be characterized by a simple dependence on wavelength. Table 1 shows the wavelength ranges covered by each small interval and the notation we will use in the following.

In order to test the theoretical MEKAL model we generated a synthetic spectrum for each wavelength interval and convolved this with the FCS instrument response using version 2.0 of the SPEX package. We used an optically thin collision-ionization-equilibrium (CIE) model with two temperature components. Each component is defined by temperature $\left(T_{1}\right.$ and $\left.T_{2}\right)$ and emission measure $\left(E M_{1}\right.$ and $\left.E M_{2}\right)$. We added a third component to represent the instrumental background which we found could be adequately represented over each spectral interval by a power law, i.e. background flux $=N_{\mathrm{pl}} E^{-\gamma}$ where $E$ is the photon energy in $\mathrm{keV}$. Values of appropriate parameters are given in Tables 2 and 3. Note that temperatures are expressed in $\mathrm{keV}(1 \mathrm{keV}=11.6 \mathrm{MK})$, emission measures in $10^{48} \mathrm{~cm}^{-3}$, and the parameter $N_{\mathrm{pl}}$ is in units of $10^{34}$ photons $\mathrm{s}^{-1} \mathrm{keV}^{-1}$ at $1 \mathrm{keV}$ (for a distance of $1 \mathrm{AU}$ ). Sometimes the first component has an unphysically low temperature $\left(T_{1} \lesssim 0.1 \mathrm{keV}\right.$ with high emission measure; in such cases, this component does not contribute significantly to the spectrum but merely serves to obtain a better statistical fit. Solar photospheric abundances (Anders \& Grevesse 1989) were assumed, except in one case (scan $\mathrm{s} 12)$ for $\mathrm{O}$ for which the relative abundance was taken to be $0.559 \times$ Anders \& Grevesse's value to fit the O viII Ly- $\alpha$ and Fe XVII lines. We assumed that the lines have profiles which are a convolution of a thermal Doppler broadening (Gaussian) profile, in which the ion temperature is equal to the corresponding electron temperature $T_{1}$ or $T_{2}$, and the instrumental profile determined by the crystal rocking curve which was assumed to be Lorentzian. We neglected any additional broadening mechanisms, e.g. due 
Table 1. Wavelength ranges of spectral intervals for spectra analyzed

\begin{tabular}{|c|c|c|c|}
\hline Spectrum Date & Scan Name $^{1}$ & Wavelength Range $(\AA)$ & FCS Channel No. \\
\hline 1980 Aug. 25 & s41 & $4.928-6.244$ & 4 \\
& s42 & $6.245-7.607$ & 4 \\
& s31 & $7.324-9.154$ & 3 \\
& s32 & $9.154-10.089$ & 3 \\
& s21 & $10.556-13.113$ & 2 \\
& s23 & $13.115-14.934$ & 2 \\
& s11 & $13.094-16.487$ & 1 \\
& $\mathrm{~s} 12$ & $16.491-22.430$ & 1 \\
\hline 1985 July 2 & $\mathrm{smm} 5$ & $7.335-7.622$ & 3 \\
& $\mathrm{smm} 1$ & $7.829-8.337$ & 3 \\
& $\mathrm{smm} 2$ & $8.504-8.665$ & 3 \\
& $\mathrm{smm} 3$ & $8.669-9.354$ & 3 \\
& $\mathrm{smm} 4$ & $9.355-10.036$ & 3 \\
\hline
\end{tabular}

${ }^{1}$ See text and Figs. 3-6. Scan s22 (range 13.115-14.908 $\AA$ ) was not used in the final analysis.

Table 2. Fit parameters of the 1980 August 25 flare spectra observed by SMM/FCS (see text for explanation of symbols)

\begin{tabular}{|lrl|ll|ll|cc|c|c|}
\hline Scan & $\chi^{2}$ & Bins & $E M_{1}$ & $T_{1}$ & $E M_{2}$ & $T_{2}$ & $N_{\mathrm{pl}}$ & $\gamma$ & $n_{\mathrm{G}}$ & Remarks \\
\hline $\mathrm{s} 11$ & 70188 & 643 & 0.881 & 0.239 & 0.952 & 0.613 & -0.910 & -10 & 0 & $\mathrm{u}$ \\
& 35421 & & 0.518 & 0.268 & 1.27 & 0.607 & -1.39 & -4.63 & 0 & $\mathrm{c}$ \\
& 21492 & & 0.769 & 0.355 & 1.04 & 0.616 & -0.915 & 0.587 & 13 & $\mathrm{cc}$ \\
\hline $\mathrm{s} 12$ & 42557 & 1027 & 0.278 & 0.1461 & 0.610 & 0.529 & 43.2 & -10 & 0 & $\mathrm{u}$ \\
& 11600 & & 5.77 & 0.0771 & 1.63 & 0.550 & 14.3 & -10 & 0 & $\mathrm{c}$ \\
& 7171 & & 1.85 & 0.0841 & 1.34 & 0.564 & 17.7 & -10 & 5 & $\mathrm{cc}$ \\
& 6186 & & 0.578 & 0.119 & 1.81 & 0.594 & 9.96 & -10 & 5 & $\mathrm{cc}$ \\
\hline $\mathrm{s} 21$ & 45226 & 1010 & 1300 & 0.0791 & 0.317 & 0.582 & 0.136 & -10 & 0 & $\mathrm{u}$ \\
& 14059 & & 12.4 & 0.140 & 0.657 & 0.564 & 0.065 & -10 & 0 & $\mathrm{c}$ \\
& 8440 & & 37.4 & 0.119 & 0.649 & 0.554 & 0.0147 & -10 & 13 & $\mathrm{cc}$ \\
\hline $\mathrm{s} 23$ & 11073 & 1030 & 0.591 & 0.211 & 0.091 & 0.619 & 0.175 & 10 & 0 & $\mathrm{u}$ \\
& 5500 & & 0.545 & 0.219 & 0.155 & 0.598 & 0.129 & 10 & 0 & $\mathrm{c}$ \\
\hline $\mathrm{s} 31$ & 4144 & 578 & $3.0110^{5}$ & 0.0854 & 3.18 & 0.640 & -0.162 & 0.734 & 0 & $\mathrm{u}$ \\
& 2180 & & $2.6410^{5}$ & 0.0867 & 4.97 & 0.583 & -1.27 & 3.93 & 0 & $\mathrm{c}$ \\
\hline $\mathrm{s} 32$ & 23158 & 885 & 59.6 & 0.128 & 0.226 & 0.564 & $-7.1210^{-3}$ & -10 & 0 & $\mathrm{u}$ \\
& 4428 & & - & - & 0.467 & 0.447 & 0.800 & 10 & 0 & $\mathrm{c}$ \\
& 3662 & & - & - & 0.493 & 0.432 & $2.8410^{-4}$ & -5.406 & 1 & $\mathrm{cc}$ \\
\hline $\mathrm{s} 41$ & 147 & 108 & 0.748 & 0.450 & 0.079 & 2.134 & 3.47 & 6.08 & 0 & $\mathrm{u}$ \\
& 140 & & 0.435 & 0.542 & 0.046 & 1.337 & 3.65 & 6.02 & 0 & $\mathrm{c}$ \\
\hline $\mathrm{s} 42$ & 989 & 295 & $6.3910^{4}$ & 0.080 & 0.196 & 0.564 & -0.0185 & 1.15 & 0 & $\mathrm{u}$ \\
& 786 & & $1.0610^{5}$ & 0.080 & 0.234 & 0.617 & -0.0182 & 0.523 & 0 & $\mathrm{c}$ \\
\hline
\end{tabular}

to plasma turbulence. Some rebinning was applied to improve the statistics of the weaker lines. A broad Gaussian component centred at $22.4 \AA$ was included to fit the instrumental background emission which greatly increases at $\lambda>20 \AA$.

Using the existing atomic data in MEKAL without any corrections, a value for $\chi^{2}$ was derived from the comparison of the synthesized spectrum with the observed data, with account taken of the photon count statistics. We repeated the process with different fit parameters until a minimum value for $\chi^{2}$ was derived. Table 2 and Table 3 give the details of the fits to the spectra of the two flares we analyzed, in which the fit parameters for this first trial are labelled by the letter " $u$ ". This did not result in a good fit, e.g., for the s11 scan of the 1980 August 25 flare $\chi^{2}=$ 70188 for 643 spectral bins (Table 2).

\subsection{Line wavelength adjustments}

In the range covered by the two flare spectra analyzed here (5-22 $\AA$ ), the MEKAL code has about 3100 lines with associated wavelengths and excitation data from various sources. For the lines from this set that occur in the $S M M$ 
Table 3. Fit parameters of SMM FCS 1985 July 2 flare spectra (see text for explanation of symbols)

\begin{tabular}{|lrc|cc|cc|cc|c|c|}
\hline Scan & $\chi^{2}$ & Bins & $E M_{1}$ & $T_{1}$ & $E M_{2}$ & $T_{2}$ & $N_{\mathrm{pl}}$ & $\gamma$ & $n_{\mathrm{G}}$ & Remarks \\
\hline smm1 & 1195 & 384 & 0.895 & 0.588 & 1.21 & 2.077 & -0.123 & $-1.3310^{-3}$ & 0 & $\mathrm{u}$ \\
& 968 & & 0.934 & 0.633 & 1.11 & 1.826 & -0.116 & $-1.7810^{-3}$ & 0 & $\mathrm{c}$ \\
& 924 & & 0.944 & 0.635 & 1.12 & 1.835 & -0.123 & $-1.3610^{-3}$ & 2 & $\mathrm{cc}$ \\
\hline smm2 & 644 & 134 & - & - & 3.96 & 2.192 & -0.264 & 0.480 & 0 & $\mathrm{u}$ \\
& 608 & & - & - & 3.81 & 2.107 & -0.249 & 0.647 & 0 & $\mathrm{c}$ \\
& 200 & & - & - & 4.08 & 2.246 & -0.437 & 0.589 & 5 & $\mathrm{cc}$ \\
\hline smm3 & 22533 & 633 & 1.84 & 0.681 & 2.48 & 3.000 & 0.145 & 0.056 & 0 & $\mathrm{u}$ \\
& 5137 & & 4.52 & 0.667 & 7.65 & 2.619 & -1.09 & 0.071 & 0 & $\mathrm{c}$ \\
& 4436 & & 4.47 & 0.663 & 6.04 & 2.386 & -0.871 & 0.071 & 6 & $\mathrm{cc}$ \\
\hline smm4 & 4590 & 411 & 6.90 & 0.302 & 0.498 & 0.903 & 0.911 & 1.62 & 0 & $\mathrm{u}$ \\
& 2445 & & 11.5 & 0.304 & 1.26 & 1.006 & 0.096 & 0.271 & 0 & $\mathrm{c}$ \\
& 1098 & & 4.33 & 0.354 & 1.31 & 1.047 & 0.035 & -0.153 & 10 & $\mathrm{cc}$ \\
\hline smm5 & 508 & 199 & - & - & 3.13 & 1.109 & -0.087 & 0.247 & 0 & $\mathrm{u}$ \\
& 299 & & 8.35 & 0.756 & 0.644 & 1.589 & -0.399 & 0.213 & 0 & $\mathrm{c}$ \\
& 275 & & 8.39 & 0.757 & 0.655 & 1.598 & -0.413 & 0.229 & 2 & $\mathrm{cc}$ \\
\hline
\end{tabular}

flare spectra with sufficient intensity we made small corrections to the wavelengths in about 450 cases to match the $S M M$ wavelengths. As will be mentioned, we also had recourse to laboratory (EBIT and PLT) data for confirmation or in some cases slight correction of the $S M M$ solar data, enabling us to make a better judgement of the wavelength shift needed.

The original line wavelengths from the MEKAL code (Mewe et al. 1995) and those after applying small shifts are given in Appendix I, along with the $S M M$ and laboratory wavelengths, intensity estimates, and transition details. The spectral interval that each line occurs in is also listed, following the notation of Table 1 . The data are given in eleven tables, each covering a particular wavelength range of the 1980 August 25 and 1985 July 2 flares. The energy level notation used in specifying the transitions in the last two columns of Tables 1 to 8 of Appendix I is that of Mewe et al. (1985) except for the ions Fe XVII to Fe XXIV where the notation is based on a convention used by one of us (D.A.L.) in calculations made with the HULLAC code. The energy level scheme is given in the seven tables making up Appendix II. The notation used by Wargelin et al. (1998) for the Fe ion lines observed from the PLT tokamak is added to the last column of Tables 9 to 11 of Appendix I.

Some second-order $(N=2)$ lines are sometimes significant. The strongest are lines of $\mathrm{Mg}$ XI at $9.170 \AA$ (appearing at $18.323 \AA$ ) and $9.315 \AA$ (at $18.612 \AA$ ). (The lines have apparent wavelengths not exactly double the true values because of crystal refractive index and flatness corrections.) Details are given in Appendix I.

As mentioned previously, the region of overlap between FCS channels 1 and 2 in the range $13.10-14.94 \AA$ for the 1980 August 25 flare gives us a means of checking some line wavelengths. For some lines, we considered the channel 1 wavelengths (from scan s11) to be more precise since the statistical quality of the observed line feature was better than for channel 2, but for other lines the better spectral resolution of channel 2 in this region (scan s23) meant that the wavelengths from this channel were more precise. As indicated already, the channel 1 scan was taken at an earlier stage in the flare decay than the channel 2 scan, a fact which can be illustrated to some extent by the two-temperature coronal models for the s11 and s23 scans that cover this overlap region; although the two temperatures $T_{1}$ and $T_{2}$ are similar, the emission measure ratio $E M_{1} / E M_{2}$ is much lower for s11, showing the presence of hotter material in the case of the 11 scan. This was taken into account when identifying lines in each range.

In the last column of Table 2 and Table 3 ("Remarks"), fits with the MEKAL code with wavelengths from Mewe et al. (1995) are labelled " $u$ " (for uncorrected) and the fits with corrected wavelengths are labelled "c". As can be seen, the fit as measured by the value of $\chi^{2}$ is significantly better. Thus, for the s11 scan $\chi^{2}$ is reduced from 70188 to 35421 (Table 2).

Apart from blends of three or more lines, the magnitudes of the shifts applied to the MEKAL wavelengths are less than or equal to $20 \mathrm{~m} \AA$ for the 1980 August 25 scans s41, s42, s31, and s21 (i.e. wavelengths less than about $13 \AA$ ), but up to $35 \mathrm{m \AA}$ for a few lines in the s11 scan and $40 \mathrm{~m} \AA$ for a very weak line in the s 12 scan. The maximum shift applied in the case of lines in the 1985 July 2 flare scans is $11 \mathrm{~mA}$. Table 4 gives values of root-mean-square (rms) wavelength differences between either the Phillips et al. (1982) or Fawcett et al. (1985) wavelengths, adjusted to the wavelength scales defined by the SSW software, and the MEKAL (Mewe et al. 1995) wavelengths. For observed line features with multiple identifications, the theoretical line with wavelength nearest to the observed wavelength is the one used to calculate the rms shift.

As well as the need for wavelength shifts in the MEKAL code, there are a number of observed line features that are either much more intense than 
Table 4. Root-mean-square wavelength differences

\begin{tabular}{|c|c|c|c|c|c|c|c|}
\hline 1980 August 25 flare scans & & & & & & & \\
Scan & $\mathrm{s} 41$ & $\mathrm{~s} 42$ & $\mathrm{~s} 31$ & $\mathrm{~s} 32$ & $\mathrm{~s} 21$ & $\mathrm{~s} 11$ & $\mathrm{~s} 12$ \\
$\sqrt{|\Delta \lambda|^{2} / N}$ & 5 & 7 & 7 & 3 & 13 & 13 & 20 \\
$N$ & 10 & 10 & 15 & 11 & 90 & 85 & 10 \\
\hline 1985 July 2 flare scans & & & & & & & \\
Scan & smm5 & $\mathrm{smm} 1$ & $\mathrm{smm} 2$ & $\mathrm{smm} 3$ & $\mathrm{smm} 4$ & & \\
$\sqrt{|\Delta \lambda|^{2} / N}$ & 3 & 6 & 4 & 7 & 5 & & \\
$N$ & 7 & 11 & 6 & 14 & 9 & & \\
\hline
\end{tabular}

$\sqrt{|\Delta \lambda|^{2} / N}$ is the root-mean-square difference (in $\mathrm{m} \AA$ ) of the $\mathrm{Ph} 82$ or Fa87 wavelengths and those in the original MEKAL code (Me95) for observed line features which are composed of not more than two identified lines. $N$ is the number of lines in each scan.
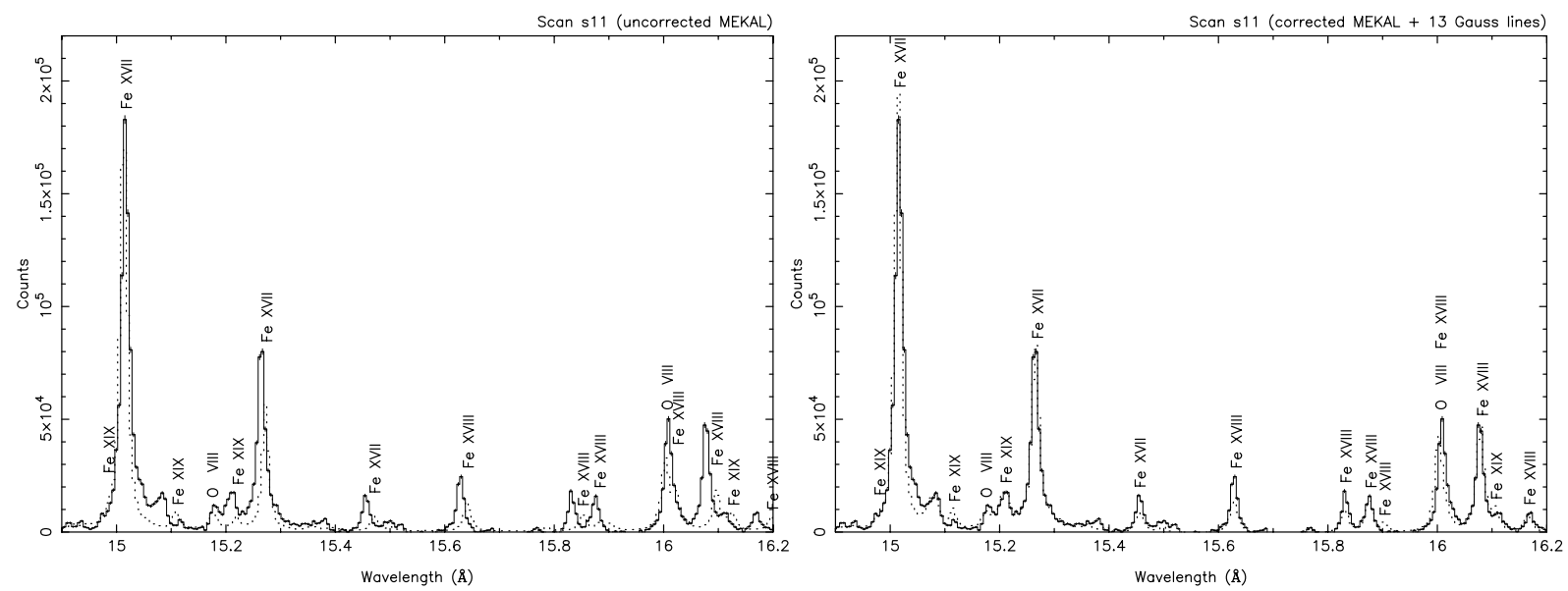

Fig. 2. Illustration of how an observed solar (SMM FCS) spectrum (part of s11 in Table 1) was fitted with the MEKAL code (dotted line) before (left panel) and after (right panel) with wavelength corrections described in the text, and with thirteen lines which were observed but apparently not included or too weakly predicted in the MEKAL code fitted with Gaussian profiles

corresponding lines in MEKAL at or near the appropriate wavelengths or are without identifications altogether. These mismatches are likely to be due to incorrectness in our emission measure model since it can be expected that the theoretical formation rates of some lines are not accurate enough to match the observed ones and since most other lines are reasonably well fitted. We have therefore attempted to improve the $\chi^{2}$ values by fitting these observed line features with Gaussian profiles; we will refer to these as "missing" lines. In SPEX we can combine a maximum of 16 different models. Thus, in addition to the two thermal components and the power-law background component, we may add a number $n_{\mathrm{G}}$, maximum 13 , of Gaussian lines. These fits are labelled "cc" in the last column of Table 2 and Table 3. For example, if we fit the s11 spectrum with the model extended by $n_{\mathrm{G}}=13$ Gaussian lines centred at the wavelength positions of the strongest missing lines, we reduce $\chi^{2}$ to 21492 (cf. Table 2). There is hence a significant further improvement in the fit.

An illustration of the improvement is shown in Figs. 2a and $2 \mathrm{~b}$. The section s11 of the 1980 August 25 flare is shown (histogram) in each of these figures with the uncorrected MEKAL fit ("u") superimposed (dotted curve) in Fig. 2a, then with the corrected MEKAL fit together with Gaussian lines added in Fig. 2b ("cc").

In Fig. 3 and Fig. 4 we present the spectra measured in the eight spectral intervals of the 1980 August 25 flare (Phillips et al. 1982) and in Fig. 5 and Fig. 6 the spectra in the five spectral intervals of the 1985 July 2 flare (Fawcett et al. 1987). In addition, part of the July 2 flare scans smm3, smm4 and smm5 are shown on an expanded wavelength scale. The figures show the SMM FCS spectra (histograms) together with the best-fit model spectra (dotted lines) using the MEKAL model with corrected line wavelengths and "missing lines" added. Identifications of the strongest lines are indicated on these figures by the emitting ion, although these are sometimes omitted where there are several contributions or the feature includes a "missing" line (e.g. at $\lambda 10.770 \AA$ ).

\subsection{Sources of error}

Despite the improvements in the fits, our spectral synthesis code would not appear to fit the high-resolution $S M M$ spectra in a statistically acceptable way for most 
of the spectral scans analyzed in the sense that the "reduced" $\chi^{2}$ (i.e. $\chi^{2}$ divided by the number of degrees of freedom which is approximately the number of spectral bins) greatly exceeds unity. This is due to inaccuracies in atomic parameters other than wavelengths, e.g. ionization fractions and excitation rate coefficients as a function of temperature, the approximation made for the emission measure distribution, and finally the fit to the instrumental background.

Ionization fractions for Fe ions as given by Arnaud \& Rothenflug (1985) are considerably different from those in the later work of Arnaud \& Raymond (1992) as a result of improved ionization and recombination rates. It is most likely that the ionization fractions of other elements are in need of revision from the currently used Arnaud \& Rothenflug (1985) data (cf. recent calculations of the ionization balance by Mazzotta et al. 1998). At present, in the current version of SPEX we use as a default Arnaud \& Raymond (1992) (ARa) for the ion fractions of Fe and Arnaud \& Rothenflug (1985) (ARo) for the other elements, but the code has the option to use ARo for Fe or to use the recent data of Mazzotta et al. (1998).

Collision strengths and rate coefficients of the Fe L lines in the 7-19 A range have been calculated by Liedahl et al. (1995) with HULLAC, including radiative and dielectronic recombination contributions. These results were recently compared with EBIT measurements of Fe XXIII and Fe XXIV spectra (Gu et al. 1999), and found to be in very good agreement. The calculations of electron impact excitation cross-sections did not include resonant excitation, which however was shown in the experiment to contribute $\leq 5 \%$ to the total line power. In the present version of MEKAL, only the strongest Fe L lines as calculated by HULLAC are included, however, and in rather a crude way, with the emissivity of a given line approximated by taking it to be that of maximum emissivity scaled to other temperatures by assuming the same temperature dependence as was used in the MEKA code for the strongest line of the same ion (Mewe et al. 1995). Therefore, the correct temperature dependence of the line emissivity away from the maximum abundance of a given ionization stage may differ somewhat from that given in the present version of MEKAL. Hence, the current excitation rates of the Fe L lines (and also of other lines) in the present MEKAL code need revision.

For most of the spectral ranges (see Tables 2 and 3), the values of $T_{1}$ and $T_{2}$ are fairly representative of the most intense lines in those ranges, so we expect the emission measure models for these ranges to be reasonably accurate. However, for some ranges (e.g. s31 and s42 in the 1980 August 25 flare), the value of $T_{1}$ is unphysically small $(<0.1 \mathrm{keV})$ and does not represent any of the lines present, but is used only to adjust to the background. The fits for these ranges are essentially singletemperature, which is reasonable in view of the small temperature range of the strongest lines present (e.g. s42 is dominated by Si XIII lines).

Possibly the most important factor in the reduced $\chi^{2}$ still being much more than unity is the imperfect fit to the instrumental background, assumed to be a power law in photon energy. Sometimes the fitted curve appreciably departs from the observed background even though the fits to the line emission can be considered to be very satisfactory. This will have the effect of increasing $\chi^{2}$, considerably so if the background is at a high level and varying in an irregular manner.

Note that this benchmark study is a very stringent one: the $S M M$ FCS spectral resolution is very high, so that even comparatively small (e.g. $>5 \mathrm{m \AA}$ ) errors in the MEKAL line wavelength data are very important for the purposes of this work. However, the typical wavelength resolution of the instruments on board Chandra and XMM is an order of magnitude larger, so such errors would not be of significance for comparison with data from these instruments.

\subsection{Laboratory measurements}

As mentioned, we compared the SMM FCS data to sets of laboratory data from the Lawrence Livermore EBIT device and the PLT tokamak. Using flat-crystal spectrometers (Beiersdorfer \& Wargelin 1994) at the EBIT facility, extensive measurements of the iron L-shell emission have been performed. EBIT has the advantage of a tunable electron beam energy making it possible to isolate one ionization stage from neighbouring ionization stages. This helps to identify the ion unambiguously producing a particular set of lines. Measurements of nearly thirty Fe XVII lines were recently reported by Brown et al. (1998). The wavelength accuracy of these measurements was about $3 \mathrm{m \AA}$ or better. EBIT measurements of the higher ionization stages of iron, Fe XVIII through Fe XXIV, are in progress. For our comparison, we used preliminary results (Beiersdorfer et al. 1996), which have a wavelength accuracy of about $7 \mathrm{m \AA}$. Results with higher accuracy are expected to become available in the future (Brown et al. 1999). The data sets from EBIT cover the line emission in the range from about 10 to $18 \AA$. Measurements from the PLT tokamak (Beiersdorfer 1988) provide line identifications in the range from 7 to $9 \AA$, e.g., for the high- $n(\lesssim 6)$ iron L-shell transitions (Wargelin et al. 1998). The wavelength accuracy of the PLT data is very high, reaching $0.1 \mathrm{m \AA}$ in some cases.

The EBIT and PLT laboratory measurements help in line identification of the $S M M$ data. They help especially in identifying the contributions from different ions to line blends. Generally there is extremely good agreement between the wavelengths of the laboratory measurements and the $S M M$ values, validating the line identification and wavelength values obtained from the solar measurements. For the Fe XVII lines in the FCS channel 1 spectrum 

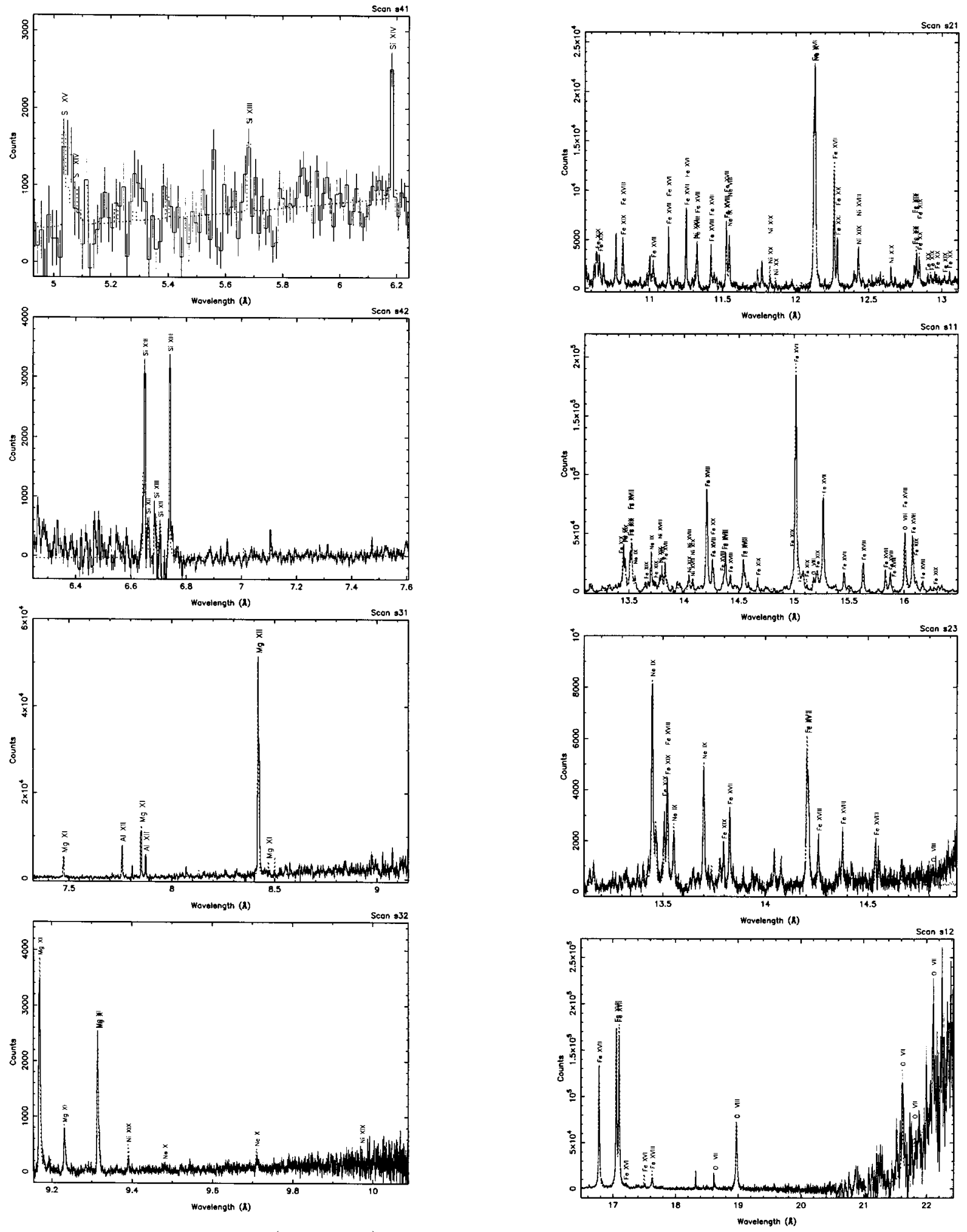

Fig. 3. Observed SMM FCS spectra (histograms) and fitted spectra using the corrected version of the MEKAL model (dotted curve) of the 1980 August 25 flare. The intensity scale ("counts") is arbitrary and should only be considered as an indication of relative intensity. Principal lines are identified with the emitting ion name. The spectral scans are (from top to bottom) s41, s42, s31, s32

Fig. 4. Observed and fitted spectra of the 1980 August 25 flare. The spectral scans are (from top to bottom) s21, s11, s23, s12. For other details, see caption to Fig. 3 

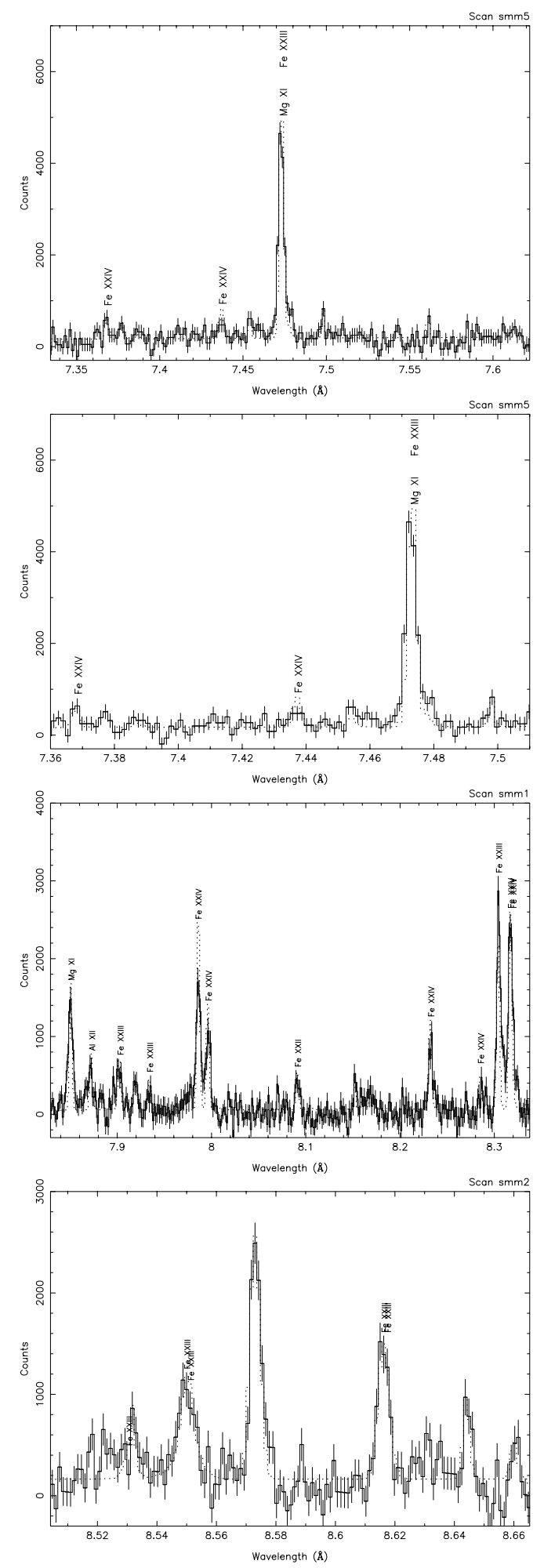

Fig. 5. Observed and fitted spectra of the 1985 July 2 flare. The spectral scans are (from top to bottom) smm5, smm5 (detail), smm1, smm2. For other details, see caption to Fig. 3
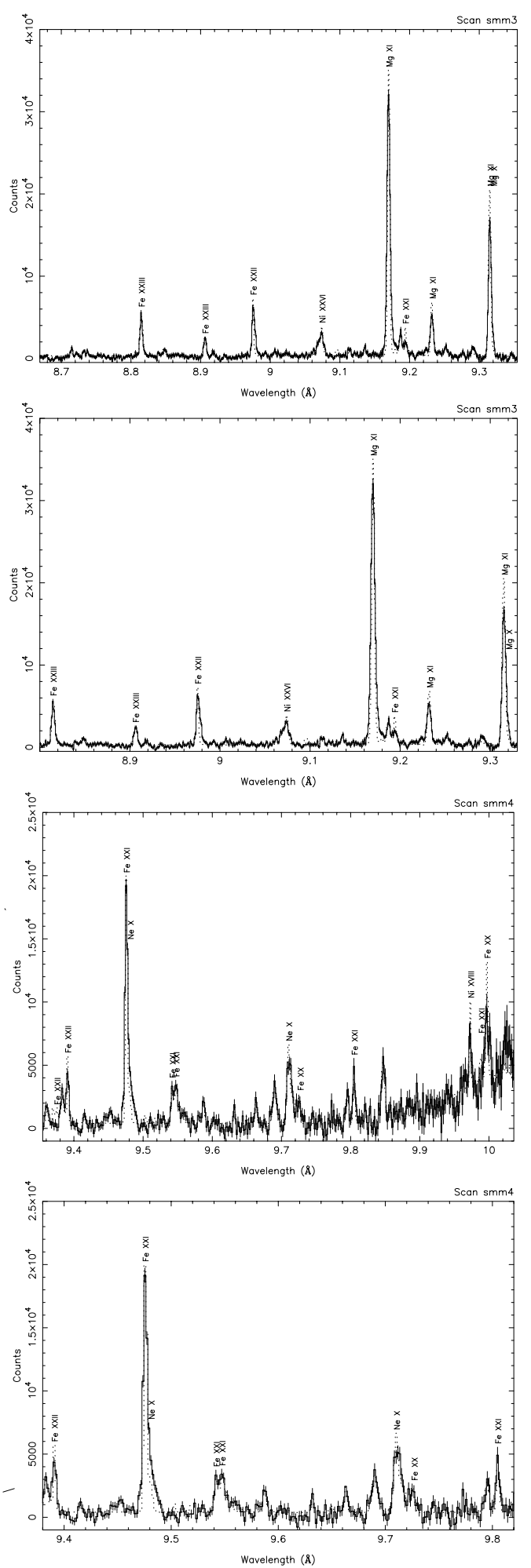

Fig. 6. Observed and fitted spectra of the 1985 July 2 flare. The spectral scans are smm3, smm3 (detail), smm4, smm4 (detail). For other details, see caption to Fig. 3 
(which has the lowest spectral resolution of the seven FCS channels) there is some tendency, as has been noted by Brown et al. (1998), for the $S M M$ wavelengths in the 1980 August 25 flare as given by Phillips et al. (1982) to be slightly less than EBIT wavelengths for this ion. However, the $S M M$ wavelengths on the wavelength scale as defined by the more recent SSW software package are up to $5 \mathrm{m \AA}$ longer, so there is now better agreement (see Appendix I). For the PLT measurements of Fe ion lines with $n=4-2$ or larger transitions reported by Wargelin et al. (1998), it is particularly noteworthy that the agreement between the $S M M$ wavelengths for the 1985 July 2 flare (Fawcett et al. 1987 ) and those from PLT is mostly to within 2 or $3 \mathrm{~mA}$. More significant differences arise where there are blends, i.e. where the composition of the solar line features cannot be clearly ascertained. We can also state the converse, namely, a significant disagreement between $S M M$ and laboratory wavelengths indicates that the solar line feature is not the same line identified in the laboratory measurements.

The analysis of the 1985 July 2 flare by Fawcett et al. (1987) established the identifications of several $n=4-2$ and $n=5-2$ transitions in Fe ions not previously published, this being done by runs of the Cowan Hartree-Fock code. Nearly all these identifications are now confirmed by the analysis of the PLT spectra (Wargelin et al. 1998). In addition, there are a number of lines which were not included by Fawcett et al. Some are present in a section of the July 2 flare spectrum from 7.34 to $7.62 \AA$ that was not analyzed in that work. A number of Fe ion lines between $7.83 \AA$ and $10.02 \AA$ which we now regard as real were ignored by Fawcett et al. because of their weakness. Finally, the intense He-like Mg (Mg XI) lines and their associated dielectronic satellites were not considered. The present analysis (given in the tables of Appendix I) includes the lines in the $7.34-7.62 \AA$ section, the previously ignored weak lines, and the $\mathrm{Mg}$ lines, and assigns identifications from the PLT spectra of Wargelin et al. (1998) and other sources.

\subsection{Identification of missing lines}

As indicated earlier, the fits to the $S M M$ FCS spectra were improved not only by adjusting line wavelengths in the MEKAL code but also by adding lines either not previously in the MEKAL code or, if present, augmenting their fluxes. This was done by fitting the line features or the required additional flux with Gaussian profiles. Most of these missing lines appear in fact to be lines already in MEKAL but whose intensities are predicted to be much smaller than observed, possibly because of inaccurate ionization fractions or excitation rate coefficients as discussed earlier.
Table 5. Comparison of wavelengths in RGS spectrum ${ }^{1}$

\begin{tabular}{|l||c|l|}
\hline Ion & $\lambda_{\text {MEKAL }}(\AA)$ & $\lambda_{\text {corr. }}(\AA)$ \\
\hline Fe XXIII & 11.761 & 11.741 \\
Fe XXII & 11.782 & 11.770 \\
Fe XVII & 12.134 & 12.124 \\
Fe XXIII & 12.184 & 12.193 \\
Fe XVII & 12.274 & 12.264 \\
Ni XIX & 12.428 & 12.430 \\
Ne IX & 13.446 & 13.448 \\
Ne IX & 13.698 & 13.700 \\
Fe XVII & 13.795 & 13.826 \\
Fe XVII & 15.272 & 15.265 \\
Fe XVII & 15.470 & 15.456 \\
Fe XVIII & 15.641 & 15.628 \\
Fe XVIII & 15.853 & 15.831 \\
Fe XVIII & 16.023 & 16.002 \\
Fe XVIII & 16.097 & 16.078 \\
Fe XVIII & 16.197 & 16.165 \\
Fe XVII & 16.796 & 16.780 \\
Fe XVII & 17.071 & 17.055 \\
Fe XVII & 17.100 & 17.119 \\
Fe XVIII & 17.664 & 17.626 \\
\hline
\end{tabular}

${ }^{1}$ cf. Fig. 7; wavelength corrections from Appendix I.

\section{Synthesized MEKAL $X M M$ Spectra}

As indicated, the shifts applied to the MEKAL line wavelengths to achieve agreement with $S M M$ flare spectra analyzed here are mostly less than $20 \mathrm{m \AA}$ for wavelengths less than $13 \AA$, but are often more significant at longer wavelengths. Some longer-wavelength lines needing substantial adjustment are very intense in SMM FCS flare and active region spectra, and can be expected to be significant in spectra of many non-solar objects to be observed by the RGS instrument on the forthcoming $X M M$ spacecraft. We illustrate the effect of MEKAL wavelength adjustments found necessary by this benchmark study by simulating a spectrum of the X-ray-emitting binary star Capella from the RGS instrument (two modules) (cf. Fig. 7). The simulated spectrum has been formed by convolving a MEKAL spectrum having the previous (plotted in Fig. 7 as solid histograms) and adjusted (dotted histograms) wavelengths with the $X M M$-RGS spectral resolution of about $50 \mathrm{m \AA}$ (FWHM), rather larger than that of the $S M M$-FCS. As a result, it is evident from the figure that the $10-20 \mathrm{m \AA}$ shifts to intense lines such as the Fe XVII lines near $12 \AA$ and $17 \AA$ will be measurable by the RGS. Another example of a perceptible difference is the comparatively large shift $(31 \mathrm{~mA})$ needed for the Fe XVII line at $13.826 \AA$, resulting in a larger separation of this line from the diagnostically important Ne IX triplet of lines near $13.4-13.7 \AA$. This example therefore shows that the applied wavelength corrections will have a strong impact on the future RGS analysis. 

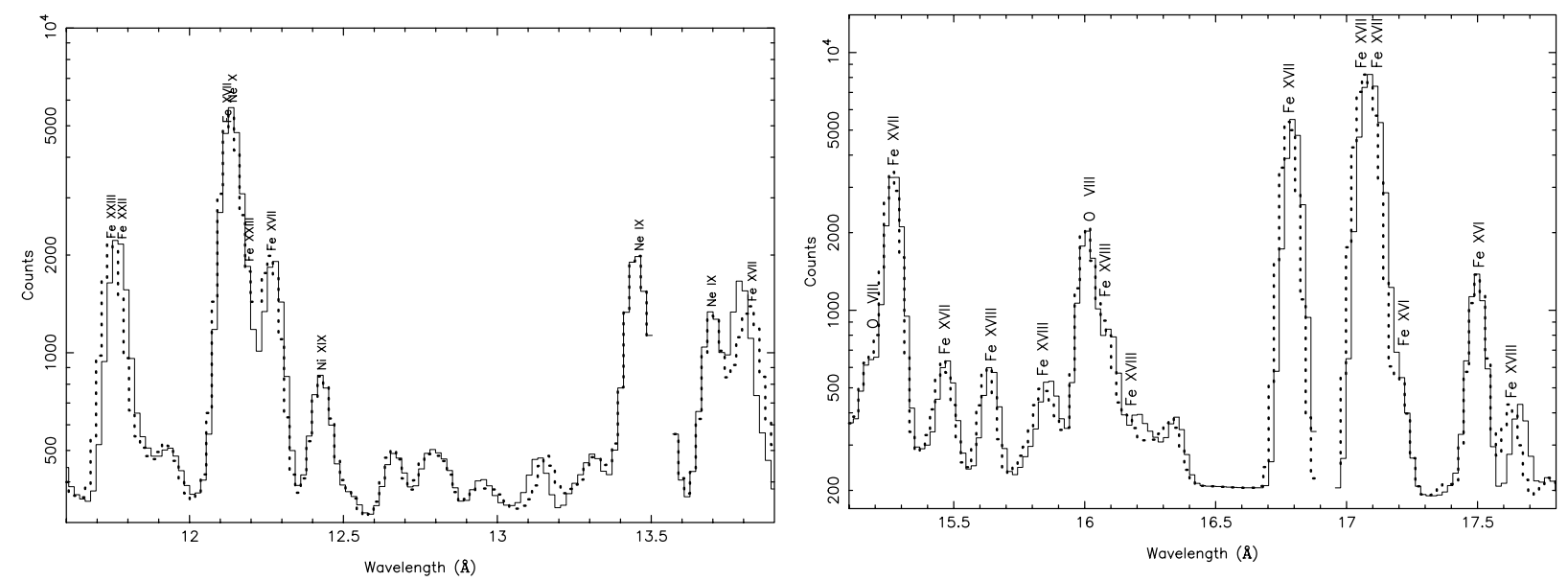

Fig. 7. Model $X M M$-RGS spectrum, plotted on a logarithmic intensity scale, of Capella simulated for 40 ks observing time using the MEKAL code with previous (histogram) and adjusted line wavelengths (dotted line with line identifications), illustrating the effects of this benchmark study

\section{Discussion and conclusions}

Our primary goal in this study was to benchmark the MEKAL spectral code using high-resolution solar flare $\mathrm{X}$-ray spectra. The two sets of solar spectra selected from the $S M M$ data are representative of a flare in a late stage of development, thus showing relatively low-excitation lines (e.g. those due to $n=3-2$ transitions in Fe ions up to Fe XXI) and an energetic flare at the peak of its development, thus showing high-excitation lines (e.g. $n=4-2$ and $n=5-2$ lines of Fe ions up to FexXIV). The comparison was made by splitting the observed spectral scans into several small scans, so that the large instrumental background varies in a simple way with wavelength.

As the wavelength precision from the $S M M$ spectra is estimated to be very high $(2-3 \mathrm{m \AA}$ for line wavelengths less than about $13 \AA$ ), the comparison with the MEKAL synthetic spectra not surprisingly showed some need for refinements in the wavelengths used in the code. Wavelength adjustments of up to $15 \mathrm{m \AA}$ were applied to the MEKAL data for lines near $15 \AA$, though most adjustments were much less than this. The statistical fits of the MEKAL spectra to the observed were consequently greatly improved (see Table 2). The value of reduced $\chi^{2}$ in fact is acceptably low for short-wavelength ranges though for longer-wavelength ranges the reduced $\chi^{2}$ remained higher than 1 . Further improvement was made by the addition of up to thirteen lines either not included or too weak in ("missing" from) the MEKAL code in each spectral range. The fact that the reduced $\chi^{2}$ is still larger than unity is probably mostly due to the poor fits to the background emission. An additional source of error is the fact that atomic data other than line wavelengths are most likely in need of revision. Future work on this is proceeding.

Figures 3 to 6 are a graphical summary of the comparison of the solar spectra and MEKAL fits, while Appendix I gives full details of the lines in the flare spectra, the MEKAL wavelengths as previously adopted (see Mewe et al. 1995), and the line identifications. Many of the lines belong to the iron L-shell complex between 7 and $17 \AA$, wavelengths of which are available from measurements of high-precision laboratory plasma spectra. The present study complements these laboratory data, since there is generally good agreement of wavelengths between the solar and laboratory data. Also, the HULLAC identifications generally used for the laboratory data are practically the same as those given by Phillips et al. (1982) and Fawcett et al. (1987) for the solar data. The only exceptions are cases of line blends in the $S M M$ data. Appendix II gives the energy level notation used in this work.

Analysis of EUVE and $A S C A$ spectra has shown that current spectral synthesis codes suffer from a number of inadequacies, but owing to the poor spectral resolution and sometimes also poor statistical quality of the observed data, it is difficult to trace the exact nature of the problems in the codes. It is likely that with the advent of the next generation of spectrometers onboard Chandra, $X M M$, and ASTRO-E, there will be a need for more accurate spectral codes to make better determinations of physical parameters, in particular temperature and emission measure. Our benchmark study in which the widely used MEKAL spectral code has been compared with solar flare spectra from the $S M M$ spacecraft having a generally high statistical quality and a spectral resolution higher than non-solar spectrometers previously flown (or even those on the future missions mentioned for wavelengths shorter than $13 \AA$ ) has provided a greatly improved spectral code. This improved code should help users to make better comparisons with future X-ray spectral data and hence obtain more reliable values of temperature, emission measure, and possibly other information for the emitting sources. 
We note that a test of the improved code will be the first analysis of Chandra data for three selected stars (Procyon, Capella, and HR 1099) as part of the so-called X-ray Emission Line Project (XELP), initiated by Nancy Brickhouse and Jeremy Drake, and to be conducted by the $A X A F$ Science Center (ASC).

\section{Further information}

The SPEX software package can be obtained via the World Wide Web, together with files containing information about installation. The WWW address of the Space Research Organisation Netherlands is http: //www.sron.nl/

in which "High Energy Astrophysics" (HEA) should be selected and then the item "SPEX". A README file and Installation Document is available together with other details. Further information may be obtained from Dr. Jelle Kaastra (e-mail J.Kaastra@sron.nl) or Dr. Rolf Mewe (R.Mewe@sron.nl).

Acknowledgements. We thank the British Council (Amsterdam) and the Netherlands Organisation for Scientific Research (NWO) for financial help with this project. KJHP and LKHM acknowledge the hospitality of SRON during their visits. Part of this work was made possible by the support of the Space Research Organization of the Netherlands (SRON). Work performed at LLNL was supported by NASA X-Ray Astronomy Research and Analysis Program work order W-19127 and performed under the auspices of the U.S. Department of Energy under contract W-7405-ENG-48. We thank Dr. F. Bely-Dubau, the referee, and Dr. S. Drake for helpful comments.

\section{References}

Anders E., Grevesse N., 1989, Geochim. Cosmochim. Acta 53, 197

Arnaud M., Raymond J., 1992, ApJ 398, 394

Arnaud M., Rothenflug R., 1985, A\&AS 60, 425

Beiersdorfer P., 1988, Ph.D. Thesis, Princeton University, \#8812108 University Microfilm, Ann Arbor, MI

Beiersdorfer P., Brown G.V., Decaux V., Kahn S.M., Liedahl D.A., Savin D.W., Widmann K., 1996, Program of the High Energy Astrophysics Division Meeting, San Diego, CA, April 29 - May 3, 1996
Beiersdorfer P., Wargelin B.J., 1994, Rev. Sci. Instrum. 65, 13 Brown G.V., Beiersdorfer P., Liedahl D.A., Kahn S.M., Widmann K., 1998, ApJ 502, 1015 (Br98)

Brown G.V., et al., 1999 (in preparation)

Cornille M., Dubau J., Loulergue M., Bely-Dubau F., Faucher P., 1992, A\&A 259, 669

Cowan R.D., 1981, The Theory of Atomic Structure and Spectra

Fabian A.C., Arnaud K.A., Bautz M.W., Tawara Y., 1994, ApJ 436, L63

Fawcett B.C., Jordan C., Lemen J.R., Phillips K.J.H., 1987, MNRAS 225, 1013

Freeland S.L., Handy B.N., 1998, Sol. Phys. 182, 497

Gu M.-F., Kahn S.M., Savin D.W., et al., 1999, ApJ (submitted)

Kaastra J.S., Mewe R., 1993, Legacy 3, 16

Kaastra J.S., Mewe R., Nieuwenhuijzen H., 1996, in: UV and X-ray Spectroscopy of Astrophysical and Laboratory Plasmas, Yamashita K., Watanabe T. (eds.). Universal Academy Press, p. 411

Klapisch M., Schwab J.L., Fraenkel J.S., Oreg J., 1977, J. Opt. Soc. Am. 61, 148

Liedahl D.A., Osterheld A.L., Goldstein W.H., 1995, ApJ 438, L115

Liedahl D.A., Osterheld A.L., Mewe R., Kaastra J.S., 1994, in: New Horizon of X-ray Astronomy - First Results from ASCA, Makino F., Ohashi T. (eds.). Universal Academy Press, Inc., p. 629

Mazzotta P., Mazzitelli G., Colafrancesco S., Vittorio N., 1998, A\&AS 133, 403

Mewe R., 1991, A\&AR 3, 127

Mewe R., Gronenschild E.H.B.M., van den Oord G.H.J., 1985, A\&AS 62, 197

Mewe R., Lemen J.R., Schrijver C.J., 1991, Astrophys. \& Space Sci. 182,35

Mewe R., Kaastra J.S., Liedahl D.A., 1995, Legacy 6, 16 (Me95)

Phillips K.J.H., 1999, in: The Many Faces of the Sun: A Summary of the Results from NASA's Solar Maximum Mission, Strong, Saba, Haisch, Schmelz (eds.), chapter 12

Phillips K.J.H., Leibacher J.W., Wolfson C.J., et al., 1982, ApJ 256, $774(\mathrm{Ph} 82)$

Phillips K.J.H., Greer C.J., Bhatia A.K., Keenan F.P., 1996a, ApJ 469, L57

Phillips K.J.H., Bhatia A.K., Mason H.E., Zarro D.M., 1996b, ApJ 466, 549

Sampson D.H., Zhang H.L., Fontes C.J., 1991, At. Data Nucl. Data Tab. 48, 25

Wargelin B.J., Beiersdorfer P., Liedahl D.A., Kahn S.M., von Goeler S., 1998, ApJ 496, 1031 (Wa98) 